\title{
Timing of Cholecystectomy for Acute Biliary Pancreatitis
}

\author{
M Mohsen Chowdhury \\ Associate Professor, Department of Surgery, Bangabandhu Sheikh Mujib Medical University, Shahbagh, Dhaka, Bangladesh
}

\begin{abstract}
Background : Biliary stones are the leading cause of acute pancreatitis. Although cholecystectomy and selective endoscopic retrograde cholangiography (ERC) comprise the current treatment in patients with acute biliary pancreatitis (ABP), the time of intervention is still controversial. Objective : In this study the outcomes of cholecystectomy was evaluated. Methods : on first admission for ABP and in patients with recurrent biliary pancreatitis. A series of 45 patients with ABP between January 2003 and November 2008 were evaluated retrospectively. Patients were classified into two groups. Group I included 30 patients who underwent cholecystectomy on first admission before discharge from the hospital. Group II comprised of 15 patients who had recurrent biliary pancreatitis and then underwent cholecystectomy. The severity of the pancreatitis was determined by Ranson's criteria. Age, gender, length of hospital stay, severity of pancreatitis, amylase level, and complications of cholecystectomy were evaluated in both groups. Patients in group I underwent cholecystectomy during the first hospital admission and patients in group II during an admission for a recurrence. Results: there were 24 patients with a Ranson's score 3 in group I and 12 in group II. The mean hospital stays were 15.29 days (range 4-48 days) and 36.66 days (range 15-123 days) in groups I and II, respectively ( $p=0.006$ ). Morbidity was $11 \%$ without mortality in group I and $43 \%$ with one mortality in group II $(p=0.023)$. Conclusions: Definitive treatment of ABP can be accomplished effectively and safely by cholecystectomy following clinical improvement, with selective ERC performed during the first admission (delayed cholecystectomy). Waiting to perform cholecystectomy (interval cholecystectomy) may result in recurrent biliary pancreatitis, which may increase morbidity and the length of the hospital stay.
\end{abstract}

Key words : Timing of cholecystectomy, acute biliary pancreatitis

[BSMMU J 2008; 1(1): 11-14]

\section{Introduction:}

Biliary calculi (stone, microlithiasis, sludge) are the leading cause of acute pancreatitis (AP). The relation between a biliary calculus and acute biliary pancreatitis (ABP) was first proposed by Opie ${ }^{1}$ in 1901 and was confirmed by Acosta and Ledsesma ${ }^{2}$ and Kelly. ${ }^{3}$ The pathogenesis of ABP involves a temporary obstruction of the ampulla of Vater by a biliary calculus migrating from the bile duct to the duodenum, causing bile reflux into the pancreas via a common channel; or when a stone is passed, the sphincter is "opened" temporarily, allowing regurgitation from the duodenum back up into the pancreatic duct ${ }^{1-2,4-5}$. Both cause AP. Evidence for this pathogenesis includes recovering stones from the stool in $86 \%{ }^{6}$ of patients with $\mathrm{ABP}$ and detecting stone impaction in the ampulla of Vater in $72 \%{ }^{7}$ of patients who underwent emergent surgery. For this etiology, although clearance of the common bile duct (CBD) and gallbladder removal were generally

Address for correspondence: Dr. M Mohsen Chowdhury, Associate Professor, Department of Surgery, BSMMU, Room no 925, C-Block, $8^{\text {th }}$ floor, email: drmohsen12@hotmail.com accepted, proper timing of the intervention was controversial before the endoscopic and laparoscopic era; and the controversy is ongoing. After the advent of endoscopic retrograde cholangiography (ERC) and endoscopic sphincterotomy (ES) for clearing the CBD, early surgery (within 24-48 hours of admission) has lost some of its importance. For cholecystectomy there are two approaches that concern proper timing: (1) interval cholecystectomy: postponing cholecystectomy 6 to 8 weeks may reduce the acute inflammation, making it easier to perform laparoscopic cholecystectomy (LC) and possibly lowering the conversion rate ${ }^{8}$; (2) delayed cholecystectomy (48 hours after admission): performing cholecystectomy during the first admission after clinical improvement may reduce the incidence of recurrent attacks of ABP, morbidity, and hospital expenses ${ }^{9}$. In this study, we evaluated the outcomes of cholecystectomy during the first admission (delayed surgery) for ABP and in recurrent biliary pancreatitis patients who underwent interval surgery after the first attack. 


\section{Methods}

\section{Patients}

A total of 45 patients with the diagnosis of ABP were evaluated retrospectively at the Department of Surgery, Hepatobiliary Division ,BSMMU,January 2003 to November 2008. The diagnosis was based on the presence of the following: (1) acute abdominal pain and tenderness; (2) elevated serum amylase level of more than $1000 \mathrm{IU} /$ L; (3) biliary calculus in the biliary tree detected by ultrasonography (US); and (4) no history of alcoholism, hypercalcemia, or lipid disorders ${ }^{9}$. Abdominal US was performed within 24 hours of admission. The severity of the disease was determined by Ransons prognostic signs 10,11: three or fewer signs indicated mild disease; more than three signs indicated severe disease. CT scan was done in 20 cases.

After the diagnosis of ABP was established, the following medical treatment modalities were employed: (1) nothing was given by mouth; (2) intravenous fluids, electrolytes, and $\mathrm{H}_{2}$-receptor blockers were administered; (3) nasogastric drainage was performed if patients had vomiting and nausea; (4) Tramadol and pethidine for pain and antibiotics for fever were administered when needed.

Patients who met one or both of the following criteria underwent early ERC and, if indicated, ES: (1) elevated serum bilirubin, alkaline phosphatase, and aspartate aminotransferase (AST) and (2) CBD diameter $>7 \mathrm{~mm}$ or US diagnosis of a biliary calculus in the CBD. Clinical improvement was determined by the serum amylase level returning to normal and alleviation of abdominal pain.

The only criterion for deciding whether patients underwent delayed or interval cholecystectomy was the surgeons preference. Patients were evaluated in two groups. Group I comprised 30 patients who underwent cholecystecyomy and early (within 72 hours) selective ERC and ES on first admission before discharge from the hospital. Group II included 15 patients who underwent selective ERC and ES during the first admission and were scheduled for interval cholecystectomy after their symptoms disappeared; 10 of these patients developed recurrent biliary pancreatitis and subsequently underwent cholecystectomy. Age, gender, length of hospital stay, severity of the pancreatitis, amylase level, and complications of cholecystectomy were evaluated in the two groups.

\section{Statistical Analysis}

Student's t-test, chi-square analysis were performed to analyze the data for significance between the two groups. Mean values (SD) were calculated. Statistical significance was defined as $p<0.05$.

\section{Results}

There were 30 patients (21 women, 9 men; mean age 54 years) in group I. Of the 15 patients who underwent interval cholecystectomy, 15 ( 8 women, 7 men; mean age 57 years) who had recurrent acute pancreatitis were included in group II. Of the 10 patients with recurrent pancreatitis, 5 had one, 3 had two, and 2 patients had three recurrent ABP attacks. The recurrence rate was $61 \%$. The groups were matched for age and gender.

The mean amylase level on admission was $2676 \mathrm{U} / \mathrm{L}$ (range 1133-5933 U/L) in group I and $3961 \mathrm{U} / \mathrm{L}$ (range 1060$8345 \mathrm{U} / \mathrm{L}$ ) in group II. Amylase level was returned to normal in 4.48 days (range 2-10 days) in group I and 3.93 days (range 2-6 days) in group II.

Biochemical analyses are shown in Table-I. Altogether, 24 patients in group I had a Ransons score of 3 or less, as did 12 in group II $(p=0.39)$.

Early selective ERC was performed in 30 patients (70\% of cases) in group I (with one failure) and 15 patients (4 during the first admission and 11 during the recurrence) (75\% of cases) in group II. Ductal stones, microlithiasis, and sludge were demonstrated in 14 patients in group I and in 8 patients in group II. All these patients underwent ES and removal of biliary calculus endoscopically.

There were three complications of sphincterotomy. In group I, One hemorrhage occurred and was managed by transfusion. Two complications occurred in group II: Pancreatitis in one patient was managed with conservative treatment, and one retroperitoneal abscess due to perforation was managed with percutaneous drainage. Both groups were matched for therapeutic ERC and its complications.

Laparoscopic cholecystectomy was performed in 22 patients in group I and in 12 patients in group II, with one conversion in each group. Morbidity after surgery was significantly higher in group II than group I, $43 \%$ and $11 \%$, respectively $(p=0.023)$. The complications of cholecystectomy are shown in Table-III.

Two patients in group II and one in group I had severe pancreatitis and underwent laparotomy for necrotizing pancreatitis and cholecystectomy. Two patient in group I had a infected pseudocyst and underwent percutaneous drainage and open cholecystectomy. One patient in group II with severe disease died owing to multiorgan failure.

The mean hospital stay was significantly longer in group II than in group I: 36.66 days (range 15-123 days) and 15.29 days (range $4-48$ days), respectively $(p=0.006$ ). The mean number of admision was 2.56 for the interval cholecystectomy group. 
Table-I

Results of biochemical analyses.

\begin{tabular}{lccc}
\hline Biochemical parameters & Group I & Group II & Normal values \\
\hline AST (U/L) & $270.0 \pm 213.84$ & $179.0 \pm 164.94$ & 038 \\
Alkaline phosphatase (U/L) & $320.0 \pm 211.85$ & $291.0 \pm 172.14$ & $39-117$ \\
Total bilirubin (mg/dl) & $3.15 \pm 3.44$ & $1.94 \pm 1.22$ & $0-1.20$ \\
\hline
\end{tabular}

AST: aspartate aminotransferase

The most common complaint of the patients on admission was abdominal pain. The patients complaints are shown in Table II.

Table-II

Patient complaints

\begin{tabular}{lcc}
\hline Complaint & $\begin{array}{c}\text { Group I (No.) } \\
(n=30)\end{array}$ & $\begin{array}{c}\text { Group II (No.) } \\
(n=15)\end{array}$ \\
\hline Abdominal pain & $30(100 \%)$ & $15(100 \%)$ \\
Nausea & $20(74.1 \%)$ & $11(68.8 \%)$ \\
Vomiting & $15(55.6 \%)$ & $10(62.5 \%)$ \\
Jaundice & $5(18.5 \%)$ & $3(18.8 \%)$ \\
\hline
\end{tabular}

Table-III

Complications of cholecystectomy.

\begin{tabular}{lcc}
\hline Complication & Group I (no.) & Group II (no.) \\
\hline Wound infection & 2 & 3 \\
Lung infection & 3 & 4 \\
\hline Total & 5 & 7 \\
\hline
\end{tabular}

\section{Discussion}

Treatment of ABP is challenging, and proper timing of any intervention is the most important factor for solving this clinical dilemma. Although procedures are similar, there are two approaches to appropriate timing of ERC and cholecystectomy ( delayed, and interval).

Before the advent of endoscopy, delayed surgery had been discussed for years. Whereas some advocated surgery for urgent biliary decompression and removal of an impacted stone from the ampulla of Vater to prevent progression of the disease ${ }^{7,12}$, others advocated delayed surgery, believing that the course of gallstone pancreatitis is not improved by early stone removal and that an impacted stone can pass into the duodenum without intervention $6,9,13$.
After the advent of ERC, the importance of early surgery has diminished, as an impacted stone can be removed and biliary decompression performed by ERC and ES. But new controversies have emerged regarding when and in whom these procedures should be performed.

It has been recommended that ERC can be performed early in patients with severe biliary pancreatitis and in whom the disease worsened or in patients with cholangitis and jaundice. ${ }^{14}$ It was shown that early ERC and ES can reduce morbidity and mortality in patients with severe $\mathrm{ABP}^{15,16}$. On the other hand, it was shown that performing ERC in all patients with ABP is unnecessary and costineffective ${ }^{17,18}$.

In this study, early elective ERC was performed in 30 patients in group I (with one failure) and in 15 patients in group II who were strongly suspected to have CBD stones based on biochemical parameters and US findings. CBD calculi were detected in 14 patients (77\%) in group I and 8 patients (66\%) in group II. The overall morbidity associated with the endoscopic procedure was $9 \%$, and there were no death.

Although the results of ERC and ES in the study correlated with those in the literature, five patients (26\% of patients who underwent ERC and ES) in group I and four patients (33\% of those who underwent ERC and ES) in group II underwent unnecessary ERC and ES, including most recent cases of this study. When the presence of a bile duct stone is strongly suspected, we prefer magnetic resonance imaging (MRI) cholangiography to avoid unnecessary ERC and are now using ERC for therapeutic purposes.

With the advent of laparoscopy and endoscopy, although early cholecystectomy is not recommended, timing of cholecystectomy following ABP is controversial. There are two approaches concerning proper timing of cholecystectomy: delayed and interval cholecystectomy. Initially, LC was not suggested in patients with $\mathrm{ABP}{ }^{19}$, but later it was concluded that LC is the preferred treatment 
with an increased rate of conversion ${ }^{8}$. Today, although some believe that LC can be performed safely and effectively as a delayed approach, except for severe ABP 20,21, others advocate interval LC following ERC ${ }^{22}$. It is thought that the interval may allow the inflammatory process to settle, but it has been shown that postponing LC is not advantageous surgically and cannot alleviate severe adhesions or avoid difficult dissection of the gallbladder, bleeding, or a prolonged operating time ${ }^{23}$.

In this study, we performed delayed LC in 22 (90\%) patients in group I and 12 (82\%) patients in group II, with one conversion in each group. It is believed that delayed LC can be performed safely in patients with ABP.

Interval cholecystectomy reportedly causes recurrent pancreatitis in $30 \%$ to $50 \%{ }^{20} ; 61 \%$ recurrence rate were observed in this study. Furthermore, five patients had one recurrent attacks, and two patients had three. Recurrent attacks cannot be prevented unless cholecystectomy is performed during the first admission.

The interval procedure was also increased the length of hospital stay and caused morbidity among this study cases. The mean hospital stay was 36.66 days in the interval cholecystectomy group and 15.29 days in the delayed cholecystectomy group. Morbidity after surgery was $43 \%$ in the interval group and $11 \%$ in the delayed group.

One patient in group I and two patients in group II had necrotizing pancreatitis and underwent laparotomy. ERC was performed in these patients before laparotomy. One of the patients in the interval group (6.2\%) died of multiorgan failure; there were no deaths in the delayed group.

\section{Conclusions}

Although it was recommended during the early 1980s to perform cholecystectomy during the first admission in patients with $\mathrm{ABP}$, a retrospective review of our records revealed that $40 \%$ of our patients underwent interval cholecystectomy. It has also been opbserved that Interval cholecystectomy may result in recurrent biliary pancreatitis, which may increase morbidity and the hospital stay. Cholecystectomy should be performed during the first admission after clinical improvement with early selective ERC in patients with ABP.

\section{References}

1. Opie EL. The etiology of acute hemorrhagic pancreatitis. Bull Johns Hopkins Hosp 1901; 12: 182-88.

2. Acosta JM, Ledsesma CL. Gallstone migration as a cause of acute pancreatitis. N Eng J Med 1974; 290: 484-487.
3. Kelly TR. Gallstone pancreatitis pathophysiology. Surgery 1976; 80: 488.

4. Kelly TR, Swaney PE. Gallstone pancreatitis: the second time around Surgery. Br J Surg 1982; 92: 571-75.

5. Acosta JM, Pellegrini CA, Skinner DB. Etiology and pathogenesis of acute biliary pancreatitis Surgery 1980; 88: 118-25.

6. Kelly TR. Gallstone pancreatitis: the timing of surgery. Surgery 1980; 88: 345-50.

7. Acosta JM, Rossi R, Galli OM. Early surgery for acute gallstone pancreatitis: evaluation of a systematic approach. Surgery 1978; 83: $367-70$

8. Tate JJ, Lau WY, Li AK. Laparoscopic cholecystectomy for biliary pancreatitis. B J Surg 1994; 81: 720-22.

9. Kelly TR, Wagner DS. Gallstone pancreatitis: a prospective randomized trial of the timing of surgery. Surgery 1988; 104: 60005.

10. Ranson JH, Rifkind KM, Roses DF et al. Prognostic signs and the role of operative management in acute pancreatitis. Surg Gyne Obstet 1974; 139: 69-81.

11. Ranson JH. Etiological and prognostic factors in human acute pancreatitis: A review. Am J Gastroenterol 1982; 77: 633-38.

12. Stone HH, Fabian TC, Dunlop WE. Gallstone pancreatitis: biliary tract pathology in relation to time of operation. Annals Surg 1981; 194: 305-12.

13. Ranson JH. The timing of biliary surgery in acute pancreatitis. Annals Surgery 1979; 189: 654-63.

14. Mergener K, Baillie J. Endoscopic treatment for acute biliary pancreatitis: when and in whom? Gastroenterol Clin North Am 1999; 28: 601-13.

15. Neoptolemos JP, Carr-locke DL, London NJ. Controlled trial of urgent endoscopic retrograde cholangiopancreatography and endoscopic sphincterotomy versus conservative treatment for acute pancreatitis due to gallstones. Lancet 1988; 2: 979-83.

16. Fan ST, Lai EC, Mok FP et al. Early treatment of acute biliary pancreatitis by endoscopic papillotomy N Eng J Med 1993; 328: 228-32.

17. Schwesinger WH, Page CP, Gross GW, et al. Biliary pancreatitis: the era of laparoscopic cholecystectomy. Arch Surg 1998; 133: 1103-06.

18. Baillie J. Treatment of acute biliary pancreatitis. N Eng J Med 1997; 336: 286-87.

19. Cuschieri A, Dubois F, Mouiel J. The European experience with laparoscopic cholecystectomy. Am J Surg 1995; 161: 385-87.

20. Delorio AV, Vitale GC, Reynolds M. Acute biliary pancreatitis: the roles of laparoscopic cholecystectomy and endoscopic retrograde cholangiopancreatography. Surg Endos 1998; 9: 39296.

21. Uhl W, Muller CA, Krahenbuhl L. Acute gallstone pancreatitis: timing of laparoscopic cholecystectomy in mild and severe disease. Surg Endos 2000; 13: 1070-76.

22. Liu CL, Lo CM, Fan ST. Acute biliary pancreatitis: diagnosis and management. World J Surg 2005; 21: 149-54.

23. Schachter $\mathrm{P}$, Peleg $\mathrm{T}$, Cohen O. Interval laparoscopic cholecystectomy in the management of acute biliary pancreatitis HPB Surgery 2007; 11: 319-23. 